\title{
Fluxo da informação científica: uma revisão dos modelos propostos na literatura em Ciência da Informação
}

\author{
Henrique Denes Hilgenberg Fernandes \\ Doutorando; Universidade de Brasília, Brasília, DF, Brasil; \\ denes@ibict.br \\ Jayme Leiro Vilan Filho \\ Doutor; Universidade de Brasília, Brasília, DF, Brasil; \\ jleiro@unb.br
}

\begin{abstract}
Resumo: Trata-se de um artigo de revisão que buscou proposições de modelos para o fluxo da informação científica documentados na literatura dos últimos 50 anos, a partir de referências das bases de dados ABCDM, BRAPCI e LISA, com o objetivo de descrever, analisar, comparar e sistematizar os achados, empregando como metodologia a pesquisa bibliográfica. $\mathrm{O}$ estudo identificou 27 modelos, considerando aspectos como sua evolução, aplicabilidade na comunicação científica (em geral ou em disciplinas ou áreas do conhecimento específicas) e abrangência geográfica dos estudos que os conceberam. Traz uma ampla análise comparativa dos modelos encontrados, no qual cada um deles é apresentado em linhas gerais, sendo descritas suas principais características, o panorama ou a aplicação para a qual foram concebidos e suas limitações. Foram estudadas ainda a influência dos modelos mais antigos sobre aqueles que os sucederam e as atualizações que alguns deles sofreram. Os modelos também foram classificados em grupos denominados síncronos, para aqueles que representam a ordem sequencial das comunicações ao longo do fluxo, ou assíncronos, para aqueles que não consideram a sequência de disseminações. Observa-se a predominância de modelos com características síncronas, destacando a importância que seus autores atribuíram à capacidade de sequenciar as comunicações e, até mesmo, quantificar os hiatos temporais existentes na cronologia do fluxo.
\end{abstract}

Palavras-chave: Comunicação científica. Produção científica. Fluxo da informação científica. Modelo de Garvey e Griffith. Unisist.

\section{Introdução}

A comunicação científica, por meio do estudo do fluxo da informação, tem utilizado modelos conceituais que representem esse fluxo como ferramentas para a descrição de fenômenos da comunicação e produção científicas, sendo 
muito empregados na fundamentação teórica de diversos trabalhos, ilustrando condições observáveis relativas aos processos de pesquisa e comunicação na ciência. Um modelo amplamente aceito é uma ferramenta importante na descrição e no estudo dos eventos relativos à comunicação científica, que podem ser adequadamente localizados e representados em regiões, funcionalidades específicas ou intervalos de tempo definidos no modelo. Também se aplica ao monitoramento e à previsibilidade da produção científica, servindo como subsídio à definição de políticas de incentivo à pesquisa, assim como à tomada de decisões por parte de gestores e pesquisadores. Por outro lado, um modelo defasado, fora de contexto ou que não represente adequadamente o fluxo da informação em um sistema de comunicação científica específico, ou ainda que não seja suficientemente aceito ou difundido, dificilmente se prestaria a representar fenômenos ou a fornecer alguma previsibilidade acerca da produção científica de forma confiável.

A partir da publicação do trabalho seminal por Garvey e Griffith (1972), a literatura tem registrado diversas outras proposições ao longo do período decorrido desde então, de forma constante e crescente, identificando uma aceleração na publicação de estudos relacionados ao tema nas últimas duas décadas (FERNANDES; VILAN FILHO, 2019). Dos modelos documentados, parte deles se propõe a descrever o fluxo da informação de forma genérica, de modo que podem ser empregados para representar todo e qualquer processo de comunicação na ciência. Outra parte desses modelos foi concebida para propósitos específicos, de forma a modelar determinadas características com um certo destaque, permitindo o estudo das condições deixadas em evidência.

Esse trabalho consistiu em uma busca em 50 anos de literatura, por meio de uma pesquisa em bases de dados brasileiras e internacionais e da leitura sistemática dos trabalhos recuperados, assim como das suas respectivas referências, com o intuito de identificar, analisar e comparar estudos que culminaram com a proposição de modelos para o fluxo da informação científica. As revisões constantes nos trabalhos correlatos encontrados quase sempre tratam de poucos modelos, geralmente os clássicos e exaustivamente explorados em livros-texto e outras fontes. Christensen (2014) aborda cinco modelos em sua 
revisão, ao passo que Khosrowjerdi (2011) traz uma comparação entre sete modelos e Gomes (2013) descreve características de dez modelos. No entanto, nenhum autor apresentou uma proposta de classificação dos modelos, tampouco os sistematizou.

A sequência desse artigo é organizada de forma que a seção 2 apresenta as definições para o fluxo da informação científica e sua conceituação nesse trabalho. A seção 3 relaciona e descreve as principais características dos modelos recuperados por meio da pesquisa bibliográfica. Análises dos trabalhos encontrados e das proposições de modelos que deles resultaram são apresentados na seção 4, e a seção 5 traz as considerações finais.

\section{0 fluxo da informação científica}

Uma forma de definir o fluxo da informação científica está relacionada com o caminho percorrido pela informação, desde o seu produtor, passando por mediadores e outros intermediários, até chegar aos seus consumidores, no caso, os usuários da informação. Sob essa ótica, Meadows (1999) define o fluxo da informação como uma maneira de ilustrar quem consulta quem, destacando, dessa forma, os gatekeepers e as fontes de informação que esses atores têm acesso. Um modelo de fluxo deve representar como se dá a transferência da informação entre os envolvidos no processo e, segundo esse autor (p. 147), “o modelo mais simples trata a transferência de informação como um processo de difusão". Esses modelos enfatizam o estudo das fontes de informação e dos diferentes canais da comunicação científica, que podem ser formais ou informais.

Para Mueller (2000), o relato oficial dos resultados de uma pesquisa concluída ocorre por meio da publicação em periódico, mas outros tipos de comunicações são também produzidos ainda durante o desenvolvimento do estudo e, também, após a sua conclusão. Essa sequência de documentos é chamada de fluxo da informação científica, que é a trajetória das sucessivas comunicações relacionadas a um projeto específico, e representa o caminho percorrido pela pesquisa desde que a ideia nasce na mente de um pesquisador, 
passando pela publicação formal dos resultados (geralmente um artigo de periódico) e continua até que a informação sobre esse artigo possa ser recuperada na literatura secundária ou apareça como citação em outros trabalhos.

Apesar de não se ter um modelo único e universal, aplicável a toda e qualquer forma de comunicação científica, a literatura traz uma série de proposições que são de grande importância para o estudo dos fenômenos que ocorrem na comunicação entre cientistas. O trabalho seminal, e talvez o mais importante, foi elaborado por Garvey e Griffith (1972), que propuseram um modelo que representa a produção de documentos relacionados a uma pesquisa ao longo de uma linha de tempo. A definição de fluxo de informação de Mueller (2000) converge totalmente para esse modelo que representa, em um projeto de pesquisa, a sequência de comunicações desde a formalização do projeto, compreendendo a fase de execução da pesquisa e estendendo-se com a publicação dos resultados, primeiramente por meio dos canais informais, passando pelos formais, finalizando com citações na literatura secundária e em trabalhos de outros autores. O modelo Unisist (UNESCO, 1971), por sua vez, ilustra a comunicação entre o produtor e o usuário do conhecimento, com ênfase nos canais, tipos de documentos e processos de mediação a que essas comunicações são submetidas. Esse modelo satisfaz plenamente a definição de Meadows por destacar as fontes de informação (agrupadas por tipos de canais), os agentes mediadores e sua atuação ao longo do fluxo.

Esses dois modelos pioneiros exemplificam de maneira perfeita as duas definições para o fluxo da informação científica, que são, de certa forma, complementares. Muitos dos modelos mais recentes são derivados desses dois trabalhos e consistem em versões aprimoradas deles.

\section{Modelos encontrados na literatura}

Foi realizado um levantamento bibliográfico, por meio de busca sistemática na literatura nas bases Arquivologia, Biblioteconomia, Ciência da Informação, Documentação e Museologia (ABCDM), Base de Dados Referenciais de Artigos de Periódicos em Ciência da Informação (BRAPCI) e Library and 
Information Science Abstracts (LISA). As buscas feitas nas bases ABCDM e BRAPCI utilizaram como argumento de pesquisa o termo "fluxo da informação científica" e, na base LISA, os argumentos "scientific information flow" e "scholarly communication flow". Os termos foram consultados nos campos palavras-chave, título e resumo. No caso específico da LISA, foram procurados ainda documentos que contivessem trabalhos de autoria de Garvey e Griffith nas suas referências em razão desses autores terem produzido o estudo seminal sobre o fluxo da informação científica e sua citação ser muito importante em trabalhos correlatos.

Os documentos correspondentes às referências desse primeiro grupo foram recuperados diretamente nas revistas ou por meio da própria base LISA e do Portal de Periódicos da CAPES. Foram também incluídos alguns trabalhos já conhecidos sobre o assunto que não constavam das referidas bases. Os documentos obtidos foram, então, analisados em ciclos incrementais de leitura. Durante a leitura daqueles artigos considerados relevantes, examinaram-se ainda outros trabalhos citados por eles, recuperando-os e acrescentando ao conjunto a ser lido. Ao todo, foram identificados 27 modelos, descritos em 18 trabalhos e resumidos aqui nas subseções que seguem, em ordem cronológica de publicação. Alguns estudos contemplam mais de um modelo, caso de Hurd (1996) e Correia (2006).

\subsection{Décadas de 1970 e 1980}

No período compreendido entre 1970 e 1989, foram identificados três estudos na literatura, os quais estão sumarizados no Quadro 1, que relaciona o nome do modelo ou o trabalho no qual esse foi apresentado e resume seus objetivos e características. O mais antigo foi o trabalho seminal de Garvey e Griffith, que estuda a sequência de comunicações produzidas, com os intervalos entre cada evento, desde o início do projeto de pesquisa, passando por instâncias da comunicação informal e semiformal, culminando com a publicação em um periódico, para depois figurar em abstracts, índices, annual reviews, citações em outros trabalhos e a inclusão dos conteúdos em tratados. Trata-se de um modelo quantitativo, concebido a partir de dados de mais de 200 projetos de pesquisa de 
associados da American Psychological Association (APA), colhidos entre 1961 e 1968.

Quadro 1 - Modelos publicados no período entre 1970 e 1989

\begin{tabular}{|l|l|}
\hline \multicolumn{1}{|c|}{ Modelo } & \multicolumn{1}{|c|}{ Características, objetivo e limitações } \\
\hline $\begin{array}{l}\text { Unesco (1971)- } \\
\text { Unisist }\end{array}$ & $\begin{array}{l}\text { Representa a comunicação científica e tecnológica entre o produtor e o } \\
\text { usuário do conhecimento. Considera canais distintos para a } \\
\text { comunicação formal, informal e tabular. Estuda as fontes de } \\
\text { informação. } \\
\text { Garvey e Griffith } \\
(1972)\end{array}$ \\
$\begin{array}{l}\text { Ilustra o fluxo da informação desde o início do projeto de pesquisa, } \\
\text { passando pelas publicações informais, a publicação em periódico, a } \\
\text { citação em annual reviews e a primeira citação por outros autores. } \\
\text { Propõe um modelo conceitual, baseado num projeto de pesquisa } \\
\text { hipotético, com 24 meses de duração e que recebe auxílio do Governo } \\
\text { dos Estados Unidos. Mostra a disseminação da pesquisa por meio de } \\
\text { canais informais e de fontes primárias e secundárias de informação. }\end{array}$ \\
\hline
\end{tabular}

Fonte: Dados da pesquisa.

O fluxo definido no modelo de Garvey e Griffith inicia quando o pesquisador já tem um problema de pesquisa bem definido, ao ponto de iniciar o planejamento da sua investigação, fase que interage com seus colegas mais próximos para discutir metodologia, análise de dados e outras questões de pesquisa. Se não receber estímulo e encorajamento de seus pares, o modelo prevê a tendência de abandonar ou procrastinar o projeto (GARVEY; GRIFFITH, 1972, p. 128). Entre seis meses e um ano do início da pesquisa, são comunicados os resultados preliminares, por meio de relatos informais, como colóquios ou reuniões do agente financiador. Entre 12 e 18 meses, segue-se a apresentação em uma conferência regional de audiência potencialmente ampla e, após 18 meses, ocorre a apresentação na reunião da APA, importante veículo que precede a comunicação em periódico.

Durante a fase de comunicações informais, especialmente durante as conferências, o autor recebe críticas, comentários e outras contribuições que são considerados para elaborar uma versão de manuscrito a ser submetido a um periódico científico. O modelo prevê que isso ocorra entre 18 e 24 meses após o início do projeto. Após a submissão ao periódico, o pesquisador inicia a distribuição informal de preprints. A publicação em periódico ocorre, de fato, apenas após três anos, ou ainda quatro anos, caso o artigo tenha sido rejeitado 
em uma primeira tentativa. Aos cinco anos, ocorre a citação nos annual reviews e as citações por outros autores se iniciam após sete anos. No oitavo ano, cabe uma notícia no Psychological Bulletin e, em se tratando de uma importante contribuição para o campo, aos treze anos é incorporado em tratados, livros especializados e livros-texto.

Quase ao mesmo tempo da publicação por Garvey e Griffith, em um esforço da United Nations Educational, Scientific and Cultural Organization (Unesco), em cooperação com o International Science Council (ISC), foi desenvolvido o modelo Unisist, que representa o fluxo entre o produtor e o usuário da informação, enfatizando o estudo das fontes de informação e do processo de mediação. O Unisist considera canais distintos para a comunicação formal, informal e tabular. O canal formal admite um ramo alternativo para a literatura cinzenta, que o modelo considera como "comunicações formais não publicadas" (UNESCO, 1971, p. 29) e o canal tabular é por onde são disseminados documentos complementares às pesquisas, como tabelas, atlas, dados e mídias diversas. É aplicado às Ciências Naturais e à Tecnologia.

O Unisist adota ainda os conceitos de "unidades documentais", que descrevem os principais tipos de documentos produzidos, assim como "unidades organizacionais", que são as instituições responsáveis pela mediação, armazenamento, custódia e preservação dessas informações. Para cada canal ou ramo de um canal, o modelo representa toda a trajetória da informação desde a sua origem no produtor, até o seu destino em seu consumidor final. Por exemplo, no caso do canal formal, um autor submete um manuscrito ao editor de um periódico, que será publicado caso seja aprovado no processo editorial. $\mathrm{O}$ periódico será impresso e distribuído, sendo armazenado em coleções nas bibliotecas, que por sua vez disponibilizam catálogos, guias e serviços de referências.

Na mesma década, Lancaster (1975) propõe um modelo conceitual, que se baseia num projeto de pesquisa hipotético, com suposto financiamento do Governo dos Estados Unidos e duração de 24 meses. Ele mostra a disseminação da pesquisa inicialmente por meio de canais informais e, em seguida, por canais formais, por meio de fontes primárias e secundárias, enfatizando os hiatos 
temporais existentes entre as fases do desenvolvimento da pesquisa e a publicação dessas fontes, demonstrando assim que a literatura periódica não é corrente. O modelo estima que, assim que o projeto de pesquisa é iniciado, o colégio invisível do qual o pesquisador é membro já tem acesso às comunicações informais. As agências de fomento costumam exigir relatórios com periodicidade trimestral, e o primeiro relatório com resultados da pesquisa é apresentado em conferência após 12 meses. Logo após, preprints e atas da conferência já estão disponíveis. Os resumos da conferência são liberados por volta do décimo quinto mês e há uma "nota prévia" em periódico aos 18 meses. Um segundo trabalho em conferência é apresentado aos 24 meses, ao mesmo tempo em que o primeiro trabalho é incluído em serviços de indexação e resumos. O primeiro artigo de periódico surge aos 30 meses e é citado no Science Citation Index (SCI) aos 40 meses. Resultados no formato monográfico, como teses e relatórios, são publicados aos 42 meses.

Nos anos que se seguiram, de 1976 a 1989, foram encontrados na base LISA apenas dois registros referentes a artigos publicados em revistas da antiga União das Repúblicas Socialistas Soviéticas (URSS), cujos resumos indicavam alguma correlação com o tema. No entanto, não foram examinados nessa pesquisa porque estavam escritos em russo.

\subsection{A virada do século - décadas de 1990 e 2000}

O Quadro 2 traz os estudos publicados nas décadas de 1990 e 2000, período que sucedeu um interstício de mais de 15 anos sem novas proposições documentadas nas fontes utilizadas. A busca por novos modelos para o processo de comunicação científica é retomada em 1992, com uma proposição de Lievrouw (1992), que apresenta o seu Ciclo para a Comunicação Científica, destacando a forma como o novo conhecimento se populariza, sendo absorvido pela sociedade e sedimentado ao conhecimento já existente na área do seu domínio científico. O estudo foi realizado a partir entrevistas conduzidas com uma amostra de pesquisadores que atuaram num grande programa de pesquisas sobre o metabolismo de lipídeos, financiadas com recursos do National Health 
Institute (NHI), numa época em que havia grande interesse social por pesquisas sobre o colesterol e sua relação com doenças coronarianas.

Quadro 2 - Modelos publicados nas décadas de 1990 e 2000

\begin{tabular}{|c|c|}
\hline Modelo & Características, objetivo e limitações \\
\hline Lievrouw (1992) & $\begin{array}{l}\text { Propõe um Ciclo para a Comunicação Científica, composto de três } \\
\text { etapas: Concepção, Documentação e Popularização do conhecimento. }\end{array}$ \\
\hline $\begin{array}{l}\text { Hurd (1996) - } \\
\text { Garvey e Griffith } \\
\text { Modernizado }\end{array}$ & $\begin{array}{l}\text { Moderniza o modelo de Garvey e Griffith com a inclusão de } \\
\text { comunicações eletrônicas como e-mails, listservers, conferências } \\
\text { eletrônicas e E-journals. }\end{array}$ \\
\hline $\begin{array}{l}\text { Hurd (1996) - No- } \\
\text { journal Model }\end{array}$ & $\begin{array}{l}\text { Modelo onde o journal deixa de existir como unidade de distribuição, } \\
\text { que passa a ser o e-article. }\end{array}$ \\
\hline $\begin{array}{l}\text { Hurd (1996) - } \\
\text { Unvetted Model }\end{array}$ & Modelo sem revisão por pares. \\
\hline $\begin{array}{l}\text { Hurd (1996) - } \\
\text { Collaboratory Model }\end{array}$ & $\begin{array}{l}\text { Modelo no qual colégios invisíveis discutem por meios eletrônicos e } \\
\text { compartilham dados e documentos utilizando-se de recursos } \\
\text { computacionais. }\end{array}$ \\
\hline $\begin{array}{l}\text { Buck et al. (1999) - } \\
\text { Scholar's Forum }\end{array}$ & $\begin{array}{l}\text { Propõem um modelo no qual, por meio de plataforma eletrônica, gerida } \\
\text { por um consórcio de universidades, são postados preprints e realizadas } \\
\text { as atividades editoriais das revistas e conferências integrantes da } \\
\text { plataforma. }\end{array}$ \\
\hline $\begin{array}{l}\text { Hurd (2000) - } \\
\text { Modelo para } 2020\end{array}$ & $\begin{array}{l}\text { Representa a forma como a autora vislumbrava a comunicação } \\
\text { científica após duas décadas: artigo como unidade básica de } \\
\text { comunicação, publicação em periódico como resultado oficial e final } \\
\text { da pesquisa, permitindo a elaboração colaborativa do texto e mantida a } \\
\text { revisão por pares. }\end{array}$ \\
\hline $\begin{array}{l}\text { Costa (2000) - } \\
\text { Modelo Híbrido }\end{array}$ & $\begin{array}{l}\text { Com o insucesso dos modelos modernizados em representar o fluxo da } \\
\text { informação, o Modelo Híbrido propõe um fluxo parte impresso e parte } \\
\text { digital. }\end{array}$ \\
\hline $\begin{array}{l}\text { Shearer e Birdsall } \\
(2002)\end{array}$ & $\begin{array}{l}\text { Modelo para a comunicação científica no Canadá, considerando um } \\
\text { panorama de novas tecnologias, globalização, interação com } \\
\text { pesquisadores estrangeiros, altos custos de assinaturas dos periódicos e } \\
\text { cortes nos investimentos governamentais nas universidades. }\end{array}$ \\
\hline $\begin{array}{l}\text { Søndergaard et al. } \\
\text { (2003) - Unisist } \\
\text { Modernizado }\end{array}$ & $\begin{array}{l}\text { Moderniza o modelo Unisist, incorporando a ele comunicações } \\
\text { eletrônicas e suporte para lidar com as diferenças disciplinares. }\end{array}$ \\
\hline Correia (2006) & $\begin{array}{l}\text { Apresenta modelos quantitativos para os fluxos das grandes áreas de } \\
\text { Ciências Biológicas, Ciências Humanas, Ciências da Saúde, Ciências } \\
\text { Exatas e da Terra, Engenharias, Ciências Sociais Aplicadas, e } \\
\text { Linguística, Letras e Artes. }\end{array}$ \\
\hline Bjork (2007) & $\begin{array}{l}\text { O autor modela todo o processo de comunicação científica, utilizando } \\
\text { uma metodologia de modelagem de processos. }\end{array}$ \\
\hline
\end{tabular}

Fonte: Dados da pesquisa. 
O Ciclo para a Comunicação Científica de Lievrouw é formado pelas etapas de Concepção, Documentação e Popularização da pesquisa. No estágio de Concepção, a comunicação é necessariamente informal, com estruturas pequenas, geralmente limitadas a uma dúzia de pessoas. No estágio de Documentação, ocorre a apresentação de resultados em conferências e publicações de natureza formal, como artigos científicos e livros. No estágio de Popularização, que não é necessariamente atingido por todas as pesquisas, a descoberta passa a fazer parte da rotina científica ou social, é convertida em conhecimento e passa para o domínio público.

Em um segundo estudo nesse período, Hurd (1996) propõe num único trabalho quatro modelos alternativos. O primeiro deles é uma atualização do modelo de Garvey e Griffith (HURD, 1996, p. 21), com o advento das novas tecnologias (Garvey e Griffith Modernizado), em particular as comunicações eletrônicas. Nos canais informais, a autora inclui $e$-mails, discussões científicas por meio de listservers e conferências eletrônicas com anais também eletrônicos. Nos canais formais, estão aos artigos em e-journals. As citações na literatura secundária são substituídas pela indexação em bases de dados digitais e os preprints também são distribuídos por meio de bases eletrônicas. Por se tratar de um cenário possível e não de um panorama real, a autora não estima os intervalos entre as diversas formas de comunicação.

O segundo modelo proposto por Hurd (1996, p. 24) no mesmo trabalho é o No-Journal Model, no qual a unidade de distribuição de periódicos deixa de ser o fascículo e passa a ser o e-article. Nas versões impressas dos periódicos, era necessário fechar uma edição para imprimir e distribuir um novo número. As primeiras revistas que foram migradas para o formato digital continuaram seguindo o formato de distribuição em fascículos, apesar de a tecnologia permitir que um artigo seja publicado assim que o manuscrito é aceito. Essa é a proposta do No-Journal, publicar o artigo assim que aceito e, com o tempo, incluí-lo em bibliotecas digitais. Mas essa realidade não se verificou. Até hoje, os periódicos eletrônicos seguem o formato de distribuição em fascículos.

O terceiro modelo é o Unvetted Model (HURD, 1996, p. 27), sem o instituto da revisão por pares, que há tempo vem sofrendo críticas de diversos 
autores, sob alegações de que a revisão privilegia trabalhos em razão do prestígio da instituição de afiliação do autor, em detrimento de outros que não tenham perfeita adequação ao paradigma em curso. Dessa forma, Hurd prevê um modelo onde o artigo é publicado assim que o manuscrito é enviado, sem nenhuma revisão. A plataforma tecnológica permite que os leitores escrevam críticas e o autor pode enviar versões revisadas. Os leitores podem ser avisados via e-mail sempre que o autor incluir uma nova versão. No entanto, embora existam proposições que visem modernizar as formas de revisão por pares, esta continua sendo a principal forma de certificação da produção bibliográfica.

O último modelo do trabalho foi o Collaboratory Model (HURD, 1996, p. 29), desenvolvido com o propósito de levar para um espaço mais amplo aquela colaboração que existe dentro de um laboratório, com o auxílio de uma plataforma digital. Pensado para um panorama de Big Science, com diversos pesquisadores, de várias instituições e em diferentes cidades e países, o modelo prevê discussões do colégio invisível via plataforma, por meio da qual dados e documentos de pesquisa são depositados num servidor, estando sujeitos à validação e escrutínio do colégio. Quando a pesquisa é concluída, os relatos ficam disponíveis em bibliotecas digitais. Dessa forma, a revisão por pares é antecipada e trazida para o início da pesquisa, validando não apenas relatos, mas também dados.

No final da década, existia um panorama de novas tecnologias que também permitiu que arquivos eletrônicos fossem replicados e transmitidos com grande facilidade e, em resposta a isso, proprietários de material protegido por direitos autorais procuraram todas as formas possíveis de negar acesso ao conteúdo de sua propriedade a qualquer um que não fosse licenciado ou assinante ativo (BUCK et al., 1999). Dessa forma, as editoras passam a deter a única versão de uma publicação em formato digital, com risco considerável de perda definitiva desse material devido ao descarte de publicações com pouco acesso (portanto, não lucrativas), à falta de investimento na conversão de arquivos em formatos obsoletos, à mudança de propriedade ou à falência dessas empresas ou, ainda, a catástrofes. 
Nesse contexto, Buck e seus colegas (1999) propõem o Scholar's Forum, um modelo que leva em conta a preservação digital dos conteúdos, pensado para ser uma plataforma tecnológica no qual um consórcio de universidades assume as atividades editoriais de periódicos e conferências. Revistas científicas de renome editadas por sociedades profissionais, assim como conferências de prestígio são incentivadas a migrar para o Scholar's Forum. Os manuscritos ou trabalhos submetidos para os periódicos ou conferências hospedadas na plataforma ficam disponíveis numa base eletrônica de preprints. Artigos e trabalhos aceitos são disponibilizados através de revistas e anais digitais, sendo o acesso aos documentos livre e garantido a dois tipos de usuários: os anônimos, com acesso de somente leitura e os registrados, que podem deixar contribuições. O processo editorial dos periódicos é coordenado pelos editores de cada revista e o processo das conferências, pelos seus respectivos comitês de programa. O modelo considera apenas trabalhos e artigos, não levando em conta outros tipos documentais, como livros, capítulos de livros e teses. Também não há registro de nenhuma implementação desse modelo.

No ano seguinte, Hurd (2000), agregando características de cada um dos modelos apresentados em seu estudo anterior (HURD, 1996), propõe um Modelo para 2020, da forma como ela vislumbrava que seria a comunicação científica 20 anos no futuro: artigo como unidade básica de comunicação, publicação em periódico como resultado oficial e final da pesquisa, permitindo a elaboração colaborativa do texto, mantida a revisão por pares e empregando todas as tecnologias disponíveis à época. Os dados de pesquisa seriam disponibilizados via web, e o relato poderia conter links para trabalhos citados e correlatos e, também, bases de preprints permitiriam o compartilhamento temporário dos resultados preliminares. O processo de edição seria totalmente automatizado, com todas as publicações em formato eletrônico e serviços agregadores atuando como intermediários no acesso às revistas solicitadas, fornecendo mecanismos de busca e links para os artigos identificados durante a pesquisa. 
Esse modelo foi objeto de crítica por Costa (2000), que verificou que naquela época ainda havia muitas publicações em formato impresso, muitas delas periódicos, e propôs um Modelo Híbrido, parte digital e parte impresso. O modelo considera algumas modalidades de comunicação informal, conferências, preprints, periódicos e elementos da literatura secundária, como índices, por exemplo, mas negligencia outros tipos documentais, como livros, capítulos de livros, teses e dissertações.

No Canadá, por mais de uma década, a Canadian Association of Research Libraries (CARL) e a Association of Universities and Colleges of Canada (AUCC) formaram uma força tarefa, cujos trabalhos resultaram, entre outros produtos, num modelo para o fluxo da informação científica no país, proposto por Shearer e Birdsall (2002). O modelo considerava um panorama de novas tecnologias, globalização, interação com pesquisadores estrangeiros, crescentes custos das assinaturas de periódicos e cortes nos investimentos públicos nas universidades. As políticas comerciais das editoras elevavam os preços das assinaturas, pois possuíam um público cativo e não estavam sujeitas às forças regulatórias do livre mercado, pois seus títulos não eram distribuídos por concorrentes, equiparando o seu mercado a um monopólio. Ao mesmo tempo em que a demanda por títulos estrangeiros nas bibliotecas universitárias aumentava, a cobertura desses caía.

De forma a mapear esse cenário, o modelo conta com quatro grupos de atores principais: pesquisadores, que realizam a pesquisa científica; editores, que criam produtos de informação; bibliotecas, que mantêm as coleções; e usuários, que convertem essa informação em novas pesquisas, produtos e serviços públicos. O modelo também enumera fatores que atuam como forças externas com influência sobre o processo de comunicação científica: tecnologia, que afeta com profundidade os processos de publicação, armazenamento e preservação; globalização, que implica numa maior colaboração entre pesquisadores canadenses e estrangeiros; economia, que deve equilibrar custos de assinaturas com orçamento das bibliotecas; mudanças nos padrões de pesquisa e novas políticas públicas para as bibliotecas. A grande limitação desse modelo é que ele considera apenas periódicos, desconsiderando outros veículos. 
Um ano depois, Søndergaard et al. (2003) apontam deficiências do modelo Unisist, como a sua aplicação à informação científica e tecnológica, particularmente às "ciências duras", assim como a falta de suporte às particularidades nacionais, regionais e diferenças paradigmáticas dentro de uma mesma disciplina. No mesmo trabalho, propõem modernizar o modelo, com versões eletrônicas de fontes de informação que já existiam no Unisist, de forma que esse fluxo eletrônico seja representado de forma indissociável à internet e acoplado ao canal tabular. O modelo também permite a fragmentação da comunicação científica por disciplina ou domínio científico, num universo transdisciplinar. Cada área do conhecimento pode importar informações de fontes naturais (da própria observação dos fenômenos), de fontes da própria disciplina, de outras disciplinas, da mídia de massa e outras, assim como exportar produtos técnicos e culturais e também documentos que servirão de fonte de informações para a própria disciplina, para outras disciplinas ou para a mídia de massa.

No Brasil, Correia (2006) estabelece modelos quantitativos para os fluxos da informação nas grandes áreas das Ciências Biológicas, Ciências Humanas, Ciências da Saúde, Ciências Exatas e da Terra, Engenharias, Ciências Sociais Aplicadas e Linguística, Letras e Artes, a partir de dados dos pesquisadores da Universidade Federal de Pernambuco (UFPE). Os dados foram coletados a partir de enquetes e entrevistas a 127 professores da universidade, detentores de bolsa de produtividade do Conselho Nacional para o Desenvolvimento Científico e Tecnológico (CNPQ). Foram considerados trabalhos em evento, artigos resumo, artigos completos, trabalhos técnicos, relatórios de pesquisa, produtos tecnológicos, capítulos de livros e livros organizados. Para cada modelo, a autora apresenta os intervalos de tempo decorridos desde o início da pesquisa, até cada tipo de publicação e os compara com aqueles interstícios descritos por Lancaster, apurando uma redução em todos os intervalos de tempo, o que ela atribui às novas tecnologias.

Ao final da década, Bjork (2007) modela o processo de comunicação científica em sua integralidade, fazendo uso da metodologia para modelagem de processos IDEF0 (NIST, 1993). O modelo proposto foi batizado pelo autor de 
Scientific Communication Life Cycle (SCLC), sendo composto por 33 diagramas diferentes em IDEF0, dispostos em uma hierarquia de sete níveis de profundidade. É bem abrangente e leva em conta a publicação de dados de pesquisa, a preservação digital dos documentos e serviços agregadores e de busca a partir de metadados. Até mesmo detalhes como a decisão do pesquisador sobre em qual revista publicar são considerados no fluxo. Por ser bem completo, pode representar o fluxo da informação em praticamente qualquer sistema de comunicação científica.

\subsection{Proposições posteriores - a partir de 2010}

No último decênio, entre 2010 e 2019, a pesquisa encontrou mais seis trabalhos sobre o assunto, que são descritos no Quadro 3 e detalhados nos parágrafos que o seguem.

Quadro 3 - Modelos posteriores a 2010

\begin{tabular}{|c|c|}
\hline Modelo & Características, objetivo e limitações \\
\hline $\begin{array}{l}\text { Khosrowjerdi e } \\
\text { Alidousti (2010) }\end{array}$ & $\begin{array}{l}\text { Propõem um modelo para o fluxo da informação científica em } \\
\text { organizações de pesquisa aplicado ao IranDoc, mas que pode ser } \\
\text { estendido para outras instituições. }\end{array}$ \\
\hline Khosrowjerdi (2011) & $\begin{array}{l}\text { Apresenta um modelo bastante simplificado do fluxo da informação, } \\
\text { baseado no Modelo de Sistema Viável de Beer, no qual a comunicação } \\
\text { científica é tratada como um organismo inteligente. }\end{array}$ \\
\hline Melo (2014) & $\begin{array}{l}\text { Apresenta um modelo quantitativo para o fluxo da informação nas } \\
\text { áreas de Ciência da Informação no Brasil. }\end{array}$ \\
\hline Christensen (2014) & $\begin{array}{l}\text { Revisa o modelo Unisist, em particular o modelo modernizado de } \\
\text { Søndergaard et al (2003), para uma aplicação na área de História da } \\
\text { Arte na Dinamarca. }\end{array}$ \\
\hline Shehata et al. (2015) & $\begin{array}{l}\text { A partir de uma classificação proposta pelo autor, que distribui os } \\
\text { pesquisadores em três perfis (ortodoxos, heterodoxos e moderados), } \\
\text { quanto ao uso de comunicações informais, é proposto um modelo que } \\
\text { ilustra a aceitação e uso das comunicações informais, para cada perfil e } \\
\text { para cada uma das atividades da comunicação científica. }\end{array}$ \\
\hline Pinto e Costa (2018) & $\begin{array}{l}\text { Pesquisa um modelo para as comunidades científicas de Ciências } \\
\text { Sociais e Humanas na Universidade do Minho. Apura que o modelo } \\
\text { que mais se aproxima dos achados é o Modelo Híbrido (COSTA, } \\
\text { 2000), com adaptações. }\end{array}$ \\
\hline
\end{tabular}

Fonte: Dados da pesquisa.

Khosrowjerdi e Alidousti (2010) elaboraram um modelo organizacional para instituições de pesquisa, aplicado ao Iranian Research Institute for 
Information Science and Technology (IranDoc), mas que pode ser estendido para outras organizações. Trata-se de um modelo conceitual que considera as fontes de informação num panorama nacional, estudando-as desde o produtor da informação até o seu usuário final e destaca a participação do IranDoc ao longo do fluxo. Em razão do seu enfoque organizacional, os relacionamentos possíveis dentro do fluxo são estudados a partir de quatro pontos de observação: pessoa para pessoa, pessoa para organização, organização para organização e organização para pessoa.

No ano seguinte, Khosrowjerdi (2011) apresenta um outro modelo organizacional, bastante simplificado do fluxo da informação, baseado no Modelo de Sistema Viável de Beer (1985), um meta modelo trazido da cibernética e usado como ferramenta de análise em diversos campos. A partir da estrutura do sistema nervoso humano, é capaz de representar organismos dotados de inteligência própria, sendo empregado no gerenciamento de processos de organizações. Por essa perspectiva, a comunicação científica passa a ser tratada como um organismo dotado de inteligência. $\mathrm{O}$ autor do modelo reivindica que ele é independente de contexto, época e escala, e o batiza de Viable Scientific Communication Model (VSCM). O modelo é composto pelos sistemas de operação, coordenação, controle, inteligência e política. Por ser bem genérico, pode ser aplicado a diversos sistemas de comunicação científica.

No Brasil, Melo (2014) traz um modelo quantitativo para o fluxo da informação nas áreas de informação no Brasil a partir da análise da produção científica relacionada a teses de doutorado. Apesar de não trazer dados que avaliem qual a parcela de participação da pós-graduação na pesquisa científica em Ciência da Informação no Brasil, o trabalho considera a produção de pesquisadores em processo de formação, mas que são orientados por cientistas experientes. A autora analisou 99 pesquisas de doutorado, por meio de um censo realizado nos seis programas de pós-graduação brasileiros na área de Ciência da Informação que tiveram teses defendidas entre 2008 e 2010. Foram considerados trabalhos em eventos, artigos de periódicos, livros e capítulos de livros publicados nos 48 meses anteriores e posteriores à defesa. Esses tipos documentais tiveram as suas ocorrências dispostas em uma linha do tempo, na 
qual o marco zero era a data da defesa da tese. A apresentação de trabalhos é mais frequente nos seis meses que antecedem a defesa, capítulos de livros são publicados nos seis meses subsequentes à defesa, ao passo que artigos de periódico são publicados entre 12 e 18 meses e livros entre 24 e 30 meses após a defesa. Por utilizar estudos realizados por cientistas ainda em processo de formação, a pesquisa pode apresentar certo viés quanto à real representação do fluxo de informação das áreas de informação.

No mesmo ano, Christensen (2014) elabora uma aplicação do Modelo Unisist Modernizado por Søndergaard e outros (2003) voltada ao fluxo da informação científica no domínio da História da Arte na Dinamarca. Bem contextualizado ao panorama da disciplina no país, levando-se em conta as suas instituições, epistemologia e sociedade, o modelo é apresentado em três estágios, sendo dois deles em perspectivas do século $\mathrm{XX}$, sob uma ótica sincrônica, em duas diferentes situações pontuais ao longo da dimensão temporal, e um terceiro, sob uma ótica diacrônica, onde o autor considera um cenário que deveria permanecer estável ao longo do tempo. Nos dois primeiros estágios, é considerada a História da Arte pelas diretrizes curriculares do curso de História da Arte da Universidade de Copenhagen em 1968 e em 2000. No terceiro cenário, são consideradas as bases epistemológicas e teóricas que estabeleceram a disciplina, levando-se em conta o arcabouço das relações disciplinares com a arte acadêmica, a crítica da arte e a filosofia estética. Foi a única aplicação do modelo Unisist encontrada fora das ditas "ciências duras".

Um ano após, Shehata et al. (2015) propõem um modelo que ilustra o fluxo quanto ao uso de comunicações informais para três perfis de pesquisadores (ortodoxos, moderados e heterodoxos), conforme uma classificação proposta por eles. Os ortodoxos utilizam apenas a comunicação formal, evitando as diferentes formas de comunicação informal. Os moderados utilizam preferencialmente as comunicações formais, porém considerando os canais informais. Já os heterodoxos, ambos os canais. O modelo considera a aceitação das comunicações informais, por perfil, e para cada uma das atividades da comunicação científica, que os autores classificam em: busca da informação, citação, colaboração, publicação e disseminação. Foi utilizada uma abordagem 
qualitativa por meio de entrevistas semiestruturadas feitas a 40 pesquisadores, em quatro universidades, questionando os seus hábitos quanto ao uso de comunicações informais. O modelo generaliza os tipos documentais agrupandoos nas classes de comunicações formais e comunicações informais e, além das atividades da comunicação científica acima, leva em conta fatores, impressões e a dissonância cognitiva (FESTINGER, 1957) que levam os pesquisadores a adotarem um dos três comportamentos categorizados.

Por fim, Pinto e Costa (2018) adaptam o Modelo Híbrido para as comunidades científicas de Ciências Sociais e Humanas, a partir de dados de pesquisadores docentes dessas áreas na Universidade do Minho, coletados através de enquetes nos anos de 2007 e 2008. O Modelo Híbrido de Costa (2000) foi escolhido por ter sido o que mais se aproximou dos resultados obtidos. O estudo apurou que, com exceção dos periódicos, a maioria das publicações ainda se dava da forma impressa e acrescentou ao modelo comunicações publicadas em repositórios institucionais.

\section{Análise dos estudos e modelos}

Dos modelos apresentados, alguns mais antigos forneceram arcabouço para novas proposições, e essas situações são ilustradas nas figuras 1 e 2. Na primeira figura, são mostradas as proposições que evoluíram a partir do modelo de Garvey e Griffith (1972). Os modelos de Correia (2006) e Melo (2014) são considerados aqui como evoluções do Modelo de Garvey e Griffith por terem empregado uma metodologia semelhante ao trabalho original, tendo ainda identificado a sequência cronológica das comunicações em uma linha do tempo. O modelo de Garvey e Griffith Modernizado, como o próprio nome diz, é uma atualização do modelo original a partir de um panorama de novas tecnologias. $\mathrm{O}$ Modelo para 2020 (HURD, 2000) faz uso das atualizações propostas pelo modelo de Garvey e Griffith Modernizado (HURD, 1996) e ainda incorpora conceitos dos modelos Collaboratory e No-journal (HURD, 1996). O Modelo Híbrido (COSTA, 2000) foi concebido a partir de críticas ao Modelo para 2020, refletindo melhor a realidade da época. Por fim, o modelo de Pinto e Costa 
(2018) utilizou parte da estrutura do Modelo Híbrido por esse ter sido o mais próximo do panorama que os dados utilizados traziam.

Figura 1 - Evolução dos modelos semelhantes ao de Garvey e Griffith

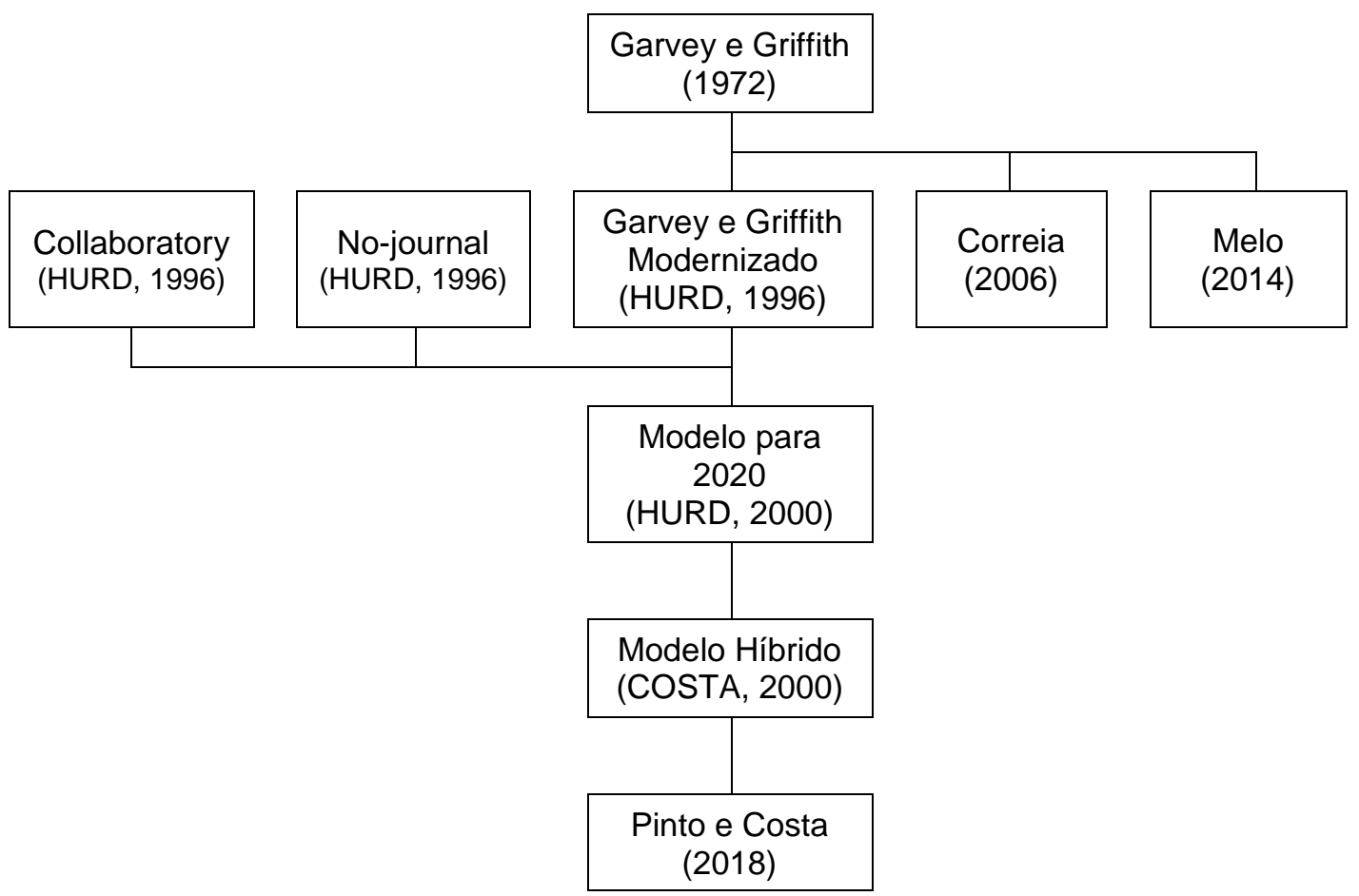

Fonte: autores.

A figura 2 mostra os modelos que evoluíram a partir do Unisist (UNESCO, 1971). Søndergaard et al. (2003) propõem atualizar o Unisist para o panorama das novas tecnologias, assim como algumas outras modificações, resultando no Unisist Modernizado. Christensen (2014) aplica o Unisist Modernizado ao domínio da História da Arte na Dinamarca.

Figura 2 - Evolução dos modelos a partir do Unisist

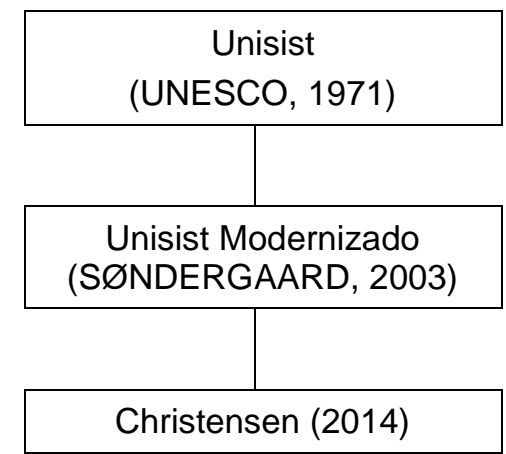

Fonte: autores. 
Alguns modelos foram concebidos em estudos limitados a uma disciplina ou área do conhecimento, como é o caso do Garvey e Griffith (1972) e outros. Muitos estudos, também, foram feitos em perímetros regionais geograficamente limitados, enquanto outros foram ainda realizados no âmbito de instituições de pesquisa. Essas limitações se deram em razão da disponibilidade dos dados utilizados nos estudos quantitativos ou por essas avaliações terem sido subsidiadas em uma conhecida estrutura da comunicação científica num espectro mais limitado. O Quadro 4, adaptado e ampliado de Fernandes e Vilan Filho (2019), resume essas características.

Quadro 4 - Modelos de fluxo de informação com delimitações geográficas ou disciplinares

\begin{tabular}{|l|c|c|c|}
\hline \multicolumn{1}{|c|}{ Modelo } & Área do conhecimento & $\begin{array}{c}\text { Delimitação } \\
\text { geográfica }\end{array}$ & Inst. \\
\hline Unesco (1971) - Unisist & Ciência e Tecnologia & - & - \\
Garvey e Griffith (1972) & Psicologia & EUA & APA \\
Lancaster (1975) & - & EUA & - \\
Lievrouw (1992) & Metab. de lipídios & EUA & NHI \\
Shearer e Birdsall (2002) & - & Canadá & - \\
Correia (2006) - Biologia & Ciências Biológicas & Recife & UFPE \\
Correia (2006) - Humanas & Ciências Humanas & Recife & UFPE \\
Correia (2006) - Saúde & Ciências da Saúde & Recife & UFPE \\
Correia (2006) - Exatas & Ciências Exatas & Recife & UFPE \\
Correia (2006) - Engenharias & Engenharias & Recife & UFPE \\
Correia (2006) - Sociais & Ciências Sociais Apl. & Recife & UFPE \\
Correia (2006) - Ling. e Artes & Ling., Letras e Artes & Recife & UFPE \\
Khosrowjerdi e Alidousti (2010) & - & Irã & IranDoc \\
Melo (2014) & Ciência da Informação & Brasil & - \\
Christensen (2014) & História da Arte & Dinamarca & - \\
Shehata & - & Reino Unido & - \\
Pinto e Costa (2018) & Ciências Soc. e Hum. & Braga & Uminho \\
\hline
\end{tabular}

Fonte: Adaptado e ampliado a partir de Fernandes e Vilan Filho (2019). Onde: APA: American Psychological Association, NHI: National Health Institute, UFPE: Universidade Federal de Pernambuco, IranDoc: Iranian Research Institute for Information Science and Technology, Uminho: Universidade do Minho

Os modelos foram ainda classificados por meio de um critério quanto à sua tipologia. A partir da definição de fluxo da informação científica de Mueller (2000), que foca nas sucessivas comunicações relacionadas a um projeto de pesquisa, com especial atenção à sequência dessas publicações e ao interstício temporal decorrido entre elas, é possível observar uma dimensão cronológica e a existência de uma linha do tempo nos modelos que satisfazem essa definição. 
Dessa forma, considera-se que todos os modelos que possuam a dimensão temporal formatada como linha do tempo, ou que pelo menos observem a sequência das comunicações, são modelos síncronos. Todos os demais são considerados assíncronos. Um exemplo de modelo síncrono é o modelo de Garvey e Griffith; e assíncrono, o Unisist. O Quadro 5 ilustra a classificação dos modelos por tipologia, demonstrando o predomínio dos modelos síncronos.

Quadro 5 - Classificação dos modelos para o fluxo da informação (síncronos/assíncronos)

\begin{tabular}{|l|l|}
\hline \multicolumn{1}{|c|}{ Síncronos } & \multicolumn{1}{c|}{ Assíncronos } \\
\hline Garvey e Griffith (1972) & \multicolumn{1}{c|}{ Unesco (1971) - Unisist } \\
Lancaster (1975) & Buck et al. (1999) - Scholar's Forum \\
Lievrouw (1992) & Shearer e Birdsall (2002) \\
Hurd (1996) - Garvey e Griffith Mod. & Søndergaard (2003) - Unisist Mod. \\
Hurd (1996) - No-journal & Khosrowjerdi e Alidousti (2010) \\
Hurd (1996) - Unvetted & Khosrowjerdi (2011) \\
Hurd (1996) - Collaboratory & Christensen (2014) \\
Hurd (2000) - Modelo para 2020 & Shehata (2015) \\
Costa (2000) - Modelo Híbrido & \\
Correia (2006) - Biologia & \\
Correia (2006) - Humanas & \\
Correia (2006) - Saúde & \\
Correia (2006) - Exatas & \\
Correia (2006) - Engenharias & \\
Correia (2006) - Sociais & \\
Correia (2006) - Ling., Letras e Artes & \\
Bjork (2007) & \\
Melo (2014) & \\
Pinto e Costa (2018) & \\
\hline
\end{tabular}

Fonte: Adaptado e ampliado a partir de Fernandes e Vilan Filho (2019).

Em relação ao método, os modelos obtidos a partir de pesquisas empíricas e quantitativas foram os de Garvey e Griffith (1972), o de Lievrouw (1992), os modelos propostos em Correia (2006), o de Melo (2014) e o de Pinto e Costa (2018). Quanto ao contexto das pesquisas, o Modelo Híbrido de Costa (2000), as proposições de Correia (2006), Shehata (2015) e Pinto e Costa (2018) foram formuladas no curso de programas formativos de pós-graduação, enquanto o trabalho de Melo (2014) foi um trabalho de conclusão de curso de graduação em Biblioteconomia. 


\section{Considerações finais}

Nesse trabalho, foram pesquisados, analisados e comparados 27 modelos de fluxo da informação científica, dos quais cinco foram obtidos de forma empírica com o emprego de métodos quantitativos, 16 surgiram de estudos limitados em termos nacionais ou regionais, 13 são limitados a disciplinas ou áreas do conhecimento e 19 deles são síncronos. As limitações regionais se deram em função da disponibilidade dos dados ou por terem sido baseados em um sistema específico e bem conhecido de comunicação científica. Os estudos mais genéricos, ou seja, que não foram limitados a uma determinada disciplina ou área do conhecimento, foram concebidos por meio de métodos qualitativos e conceituais ou ainda a partir da generalização de trabalhos anteriores.

O estudo da origem e do aperfeiçoamento das proposições identificou dois ramos principais de evolução, sendo que, no primeiro, os modelos evoluem a partir do Garvey e Griffith (1972), herdando do ancestral mais distante a característica síncrona e, no segundo ramo, evoluem a partir do Modelo Unisist (UNESCO, 1971), assimilando características assíncronas. Finalmente, a prevalência de modelos síncronos demonstra que seus autores, assim como as condições em que essas pesquisas foram elaboradas, atestam a importância da dimensão temporal, cronologicamente quantificável ou, pelo menos, a observação da sequência das comunicações.

Observou-se, também, que modelos mais genéricos, ou seja, que não são limitados a uma disciplina ou área de conhecimento, são sempre conceituais e nenhuma dessas proposições traz estudos a partir de dados quantitativos. Os modelos mais específicos ou limitados a áreas de conhecimento ou regiões são, em sua maioria, baseados em observações empíricas e em dados quantitativos ou, quando não o são, subsidiam-se em um conhecimento grande e profundo de uma área ou região estudada. Em todos os casos, esses modelos mais específicos são mais sensíveis a fenômenos e características idiossincráticas de uma determinada disciplina ou região.

Os estudos recentes encontrados demonstram que a busca por um modelo capaz de representar o fluxo da informação científica é um assunto que está longe de ser pacificado. Mudanças tecnológicas, sociais, econômicas, assim 
como diversos outros fatores influenciam o jeito de se fazer ciência e, por sua vez, a forma como essa é comunicada. Dessa forma, estudos ulteriores e constantes são importantes para investigar fenômenos relacionados e descobrir a maneira como a ciência é produzida, levando ao aperfeiçoamento constante das políticas científicas.

\section{Referências}

BEER, S. Diagnosing the System for Organization. Chichester: Wiley, 1985.

BJORK, B. C. A model of scientific communication as a global distributed information system. Information Research: an international electronic journal, Borås, v. 12, n. 2, 2007.

BUCK, A. M. et. al., Scholar's Forum: A New Model for Scholarly Communication. Pasadena, 1999. Disponível em: https://authors.library.caltech.edu/25880/2/scholarsforum.pdf. Acesso em: 14 jul. 2020.

CHRISTENSEN, H. D. The framing of scientific domains: About UNISIST, domain analysis and art history. Journal of Documentation, Bingley, v. 70, n. 2, p. 261-281, 2014.

COSTA, S. M. S. Mudanças no Processo de Comunicação Científica: O Impacto do uso de Novas Tecnologias. In: MUELLER, S. P. M.; PASSOS, E. J. L. (Orgs.). Comunicação Científica, Brasília: Departamento de Ciência da Informação, Universidade de Brasília, 2000, p. 95-105.

CORREIA, A. E. G. C. O fluxo da informação no processo de pesquisa na UFPE: as influências das tecnologias da informação e comunicação. 2006. Dissertação (Mestrado em Ciência da Informação) - Programa de PósGraduação em Ciência da Informação, Universidade Federal de Santa Catarina, Florianópolis, 2006.

FERNANDES, H. D. H.; VILAN FILHO, J. L. Fluxo da Informação Científica: uma análise dos estudos e modelos. In: ENCONTRO NACIONAL DE PESQUISA EM CIÊNCIA DA INFORMAÇÃO, 20., 2019, Florianópolis. Anais [...] Florianópolis: Universidade Federal de Santa Catarina, 2019.

FESTINGER, L. A Theory of Cognitive Dissonance. Stanford: Stanford University Press, 1957.

GARVEY, W. D.; GRIFFITH, B. C. Communication and information processing within scientific disciplines: Empirical findings for Psychology. Information Storage and Retrieval, Elmsford, v. 8, n. 3, p. 123-136, 1972. 
GOMES, C. M. Comunicação Científica: alicerces, transformações e tendências. Covilhã: Livros Labcom, 2013.

HURD, J. M. Models of Scientific Communications Systems. In: CROWFORD, S. Y.; HURD, J. M.; WELLER, A. C. (Orgs.). From Print to Electronic: the transformation of scientific communication. Medford: ASIS, 1996. p. 9-33.

HURD. J. M. The Transformation of Scientific Communication: a model for 2020. Journal of the American Society for Information Science, New York, v. 51, n. 14, p.1279-1283, 2000.

KHOSROWJERDI, M.; ALIDOUSTI, S. Scientific information transfer: A conceptual model for scientific communication in IranDoc. The Electronic Library, Bingley, v. 28, n. 6, p. 818-828, 2010.

KHOSROWJERDI, M. Designing a viable scientific communication model: VSM approach. Library Hi Tech, Bradford, v. 29, n. 2, p. 359-372, 2011.

LANCASTER, F. W. Acessibilidade da informação na pesquisa científica em processo. Ciência da Informação, Rio de Janeiro, v. 4, n. 2, p. 109-117, 1975.

LIEVROUW, L. A. Communication, representation, and scientific knowledge: a conceptual framework and case study. Knowledge and Policy, New Brunswick, v. 5, n. 1, p. 6-28, 1992.

MEADOWS, A. J. A comunicação científica. Brasília: Briquet de Lemos/Livros, 1999.

MELO, B. K. S. B. Fluxo da comunicação científica na área de Ciência da Informação no Brasil: análise da produção científica relacionada com teses defendidas de 2008 a 2010. 2014. Trabalho de Conclusão de Curso (Biblioteconomia) - Universidade de Brasília, Brasília, 2014.

MUELLER, S. P. M. A ciência, o sistema de comunicação científica e a literatura científica. In: CAMPELLO, B. S.; CENDÓN, B. V.; KREMER, J. M. (Orgs.). Fontes de Informação para Pesquisadores e Profissionais, Belo Horizonte: Ed. UFMG, 2000. p. 21-34.

NIST - National Institute of Standards and Technology. Integration definition for function modeling (IDEF0). Draft federal information processing standards publication 183, Gaithersburg: National Institute for Standards and Technology, 1993.

PINTO, C. S.; COSTA, J. L. Padrões de comunicação em diferentes comunidades científicas. In: COSTA, S. M. S.; LEITE, F. C. L.; TAVARES, R. B. (Orgs.). Comunicação da informação, gestão da informação e gestão do conhecimento. Brasília: Ibict, 2018. p. 145-159. 
SHEARER, K.; BIRDSALL, B. The transition of scholarly communications in Canada. Canada, 2002. Disponível em:

https://www.semanticscholar.org/paper/The-Transition-of-ScholarlyCommunications-in-the-Shearer-

Birdsall/ebca8f87ea2a28a6e289ce94e5a67055ec7d02ba. Acesso em: 20 jul. 2020.

SHEHATA, A. et al. The impact of information and communication technologies on informal scientific communication. Library Review, Bradford, v. 64, n. 6, p. 428-445, 2015.

SØNDERGAARD, T. F. et al. Documents and the communication of scientific and scholarly information: Revising and updating the UNISIST model. Journal of Documentation, London, v. 59, n. 3, p. 278-320, 2003.

UNESCO - United Nations Educational, Scientific and Cultural Organization. Unisist: Study Report on the Feasibility of a World Science Information System. Paris: Unesco, 1971.

\title{
Scholarly communication flow: a review of the propositions in the literature in Information Studies
}

\begin{abstract}
This review article reports propositions of models to the scholarly communication flow found in the ABCDM, BRAPCI and LISA databases, in the later 50-year literature, in order to describe, analyze, compare and systematize the findings, using bibliographic research as methodology. This study has identified 27 models, considering features as its evolution, usage in the scientific communication (general or specific to some academic subjects or areas of knowledge) and the geographical coverage of the studies that conceived it. The results consist of a wide comparative analysis of the models found, where is presented each one and are described their main features, sceneries or applications to which they were designed as well as their limitations. It also studied the influence of older models on their successors and modernized ones. These models were classified in groups named synchronous, to those that represent a sequence in the communications along the flow, or asynchronous, to those that do not consider the sequence of disseminations. It is notable the predominance of synchronous models, highlighting the importance that their authors gave to the ability of sequencing communications and even quantify time intervals existing in the flow chronology.
\end{abstract}

Keywords: Scientific communication. Scientific production. Scholarly communication flow. Model of Garvey and Griffith. Unisist. 


\section{Declaração de autoria}

Concepção e elaboração do estudo: Henrique D. H. Fernandes, Jayme L. Vilan Filho.

Coleta de dados: Henrique D. H. Fernandes

Análise e discussão de dados: Henrique D. H. Fernandes

Redação e revisão do manuscrito: Henrique D. H. Fernandes, Jayme L. Vilan Filho.

\section{Como citar}

FERNANDES, Henrique Denes Hilgenberg; VILAN FILHO, Jayme Leiro. Fluxo da informação científica: uma revisão dos modelos propostos na literatura em Ciência da Informação. Em Questão, Porto Alegre, v. 27, n. 2, p. 138-163, abr./jun. 2021.

Doi: http://dx.doi.org/10.19132/1808-5245272.138-163 